\title{
On the Structure of Zonal Spherical Functions on Symmetric Spaces of Negative Curvature of Type AII
}

\section{I. Inozemtsev}

A purely algebraic method is proposed for the construction of zonal spherical functions (ZSF) on symmetric spaces $X_{n}^{-}=S L(n, Q) / S p(n)$ and eigenfunctions of the hyperbolic Sutherland operator connected with them. Examples of the explicit calculations of the coefficients determining the structure of ZSF are given.

Keywords: hyperbolic Sutherland operator, permutation group, zonal spherical functions

\section{Introduction}

The purpose of this paper is to find an effective algorithm for constructing zonal spherical functions (ZSF) on symmetric spaces $X_{n}^{-}$of rank $n-1$ and type AII [1], realized as varieties of adjacent classes $S L(n, Q) / S p(n)$, in the cases $n \geqslant 3$. Numerous examples of ZSF on the spaces of rank 1 which are used for the calculation of matrix elements of representations of the group of rotations of pseudo-Euclidean space are given in [2].

The constructions of intertwining operators which allow one to find ZSF for the space of rank 2 of type A III $=S U(p, q) / S(U(p) \times U(q))$ were described in $[3,4]$. ZSF on the space $X_{3}^{-}$were first calculated in [5]. Up to now, for the spaces $X_{n}^{-}$of higher ranks only integral representations of ZSF have been known. They contain $2 n(n-1)$-multiple integrals on the elements of lower triangle $n \times n$ matrices constructed from quaternions [6]. An equivalent approach allows one to get the ZSF as decompositions into infinite $n(n-1) / 2$-dimensional series with coefficients defined recurrently [1]. Both these representations are of great value for the investigation of various asymptotics of the ZSF. But they are not effective in practical calculations even for the simplest case $n=3$. The use of ZSF of the spaces of higher ranks for the solution of problems of mathematical physics is caused by the following fact: under the choice of orispherical coordinate system on $X_{n}^{-}$the radial part of the quadratic Laplace-Beltrami operator can be presented in

Received March 17, 2019

Accepted June 14, 2019

Vladimir I. Inozemtsev

inozv@theor.jinr.ru

Laboratory of Theoretical Physics, JINR

ul. Universitetskaya 19, Dubna, 141980 Moscow Region, Russia

RUSSIAN JOURNAL OF NONLINEAR DYNAMICS, 2019, 15(2), 179-186 
the form $[7]$

$$
B_{n}=a^{-2}\left[\Delta_{n}+4 a \sum_{j>s}^{n} \operatorname{coth}\left[a\left(x_{j}-x_{s}\right)\right]\left(\frac{\partial}{\partial x_{j}}-\frac{\partial}{\partial x_{s}}\right), \quad x \in R^{n}, a>0,\right.
$$

where $\Delta_{n}$ is the Laplace operator in $R^{n}$. Zonal spherical functions $\Phi_{n}^{(k)}(x)$ are simply eigenfunctions of $B_{n}$ which correspond to the eigenvalues

$$
b_{n}(k)=-a^{-2}\left[k^{2}+\frac{4 n\left(n^{2}-1\right) a^{2}}{3}\right], \quad k \in R^{n} \quad\left(k^{2}=\sum_{j=1}^{n} k_{j}^{2}\right),
$$

They should be symmetric under permutations of the arguments $\left\{x_{j}\right\}$ and normalized by the condition $\Phi_{n}^{(k)}(0)=1$. Of the greatest interest for applications are the wave functions of the hyperbolic Sutherland problem which describe the processes of scattering of $n$ quantum Bose particles on the line with asymptotical momenta $k_{1}, \ldots k_{n}$ and the total energy $\epsilon(k)=k^{2} / 2$,

$$
\psi_{n}^{(k)}(x)=\prod_{j>s}^{n} \frac{\sinh ^{2}\left[a\left(x_{j}-x_{s}\right)\right]}{a^{2}} \Phi_{n}^{(k)}(x) .
$$

The singular transformation defined by (1) transfers $B_{n}$ into the Sutherland Hamiltonian

$$
H_{S}^{(n)}=-\frac{\Delta}{2}+\sum_{j>s}^{n} \frac{a^{2}}{\sinh ^{2}\left[a\left(x_{j}-x_{s}\right)\right]} .
$$

The discrete analog of $H_{S}^{n}$ is used for description of the interactions of quantum s=1/2 spins in the one-dimensional models of ferromagnetism [9].

\section{The method of calculation of the ZSF}

We propose an algorithm for constructing the functions $\psi_{n}^{(k)}(x)$ and $\Phi_{n}^{(k)}(x)$ based on the solutions of the system of linear algebraic equations. This algorithm also allows one to establish their connection with homogeneous polynomials in variables $y_{j}=\exp \left(2 a x_{j}\right)$. It is based on the result of the paper [8] in which the recurrence scheme of calculating eigenfunctions of the operator (2) was constructed,

$$
\psi_{n}^{(k)}(x)=D_{n} \exp \left(i \sum_{s=1}^{n} k_{s} x_{s}\right)
$$

where

$$
\begin{gathered}
D_{n}=Q_{n}^{1 \ldots n-1} D_{n-1}, \\
Q_{N}^{i_{1} \ldots i_{l}}=Q_{N}^{i_{1} \ldots i_{l-1}}\left[\frac{\partial}{\partial x_{i_{l}}}-\frac{\partial}{\partial x_{n}}-2 a \operatorname{coth} a\left(x_{i_{l}}-x_{n}\right)\right]+ \\
+\sum_{s=1}^{l-1} 2 a^{2} \sinh ^{-2}\left[a\left(x_{i_{s}}-x_{i_{l}}\right)\right] Q_{N}^{i_{1} \ldots i_{s-1} i_{s+1} \ldots i_{l-1}}, \quad Q_{N}=1, \\
1 \leqslant i_{1}, \ldots i_{l}<N, \quad 1 \leqslant N \leqslant n .
\end{gathered}
$$


The zonal spherical function $\Phi_{n}^{(k)}(x)$ is connected with $\psi_{n}^{(k)}(x)$ by the relation

$$
\Phi_{n}^{(k)}(x)=\mathrm{const}\left(\prod_{j>s} \frac{a^{2}}{\sinh ^{2} a\left(x_{j}-x_{s}\right)}\right) \sum_{P \in \pi_{n}} \psi_{n}^{(k)}(P x),
$$

here $\pi_{n}$ is the group of permutations of numbers $1, \ldots n$ and $P x$ is the point in $R^{n}$ with coordinates $\left(x_{P 1}, \ldots x_{P n}\right)$.

The calculations of ZSF under the scheme (3)-(4) are extremely cumbersome due to the presence of differentiation operators in the recursive relations and were in fact performed only for the case $n=3$. Below we shall show that the problem of determining $\Psi_{n}^{(k)}(x)$ admits a purely algebraic formulation.

Proposition 1. The function $\psi_{n}^{(k)}(x)$ can be represented as

$$
p\left(k,\left\{\operatorname{coth} a\left(x_{j}-x_{s}\right)\right\}\right) \times \exp \left(i \sum_{s=1}^{n} k_{s} x_{s}\right),
$$

where $p$ is a polynomial in the variables $\left\{\operatorname{coth} a\left(x_{j}-x_{s}\right)\right\}, j>s$.

The proof is based on (3), (4) and the possibility of representing $\left(\frac{d}{d z}\right)^{l}(\operatorname{coth} z)^{m}$ in the form of a polynomial of degree $l+m$ in $\operatorname{coth} z$.

As a consequence, the function

$$
\exp \left(-i \sum_{s=1}^{n} k_{s} x_{s}\right) \psi_{n}^{(k)}(x)
$$

has the period $\pi i a^{-1}$ on each argument $x_{1}, \ldots x_{n}$ and remains finite as $x_{j} \rightarrow \pm \infty, \quad 1 \leqslant j \leqslant n$. In the neighborhood of hyperplanes $x_{j}=x_{s}$ this function has a singularity of type $\left[\sinh a\left(x_{j}-\right.\right.$ $\left.\left.-x_{s}\right)\right]^{-1}$ in accordance with the structure of the Sutherland operator (2). These properties, together with Proposition 1, allow us to formulate the following

Proposition 2. $\psi_{n}^{(k)}(x)$ has a representation in the form

$$
\psi_{n}^{(k)}(x)=\left[\prod_{j>s}^{n} \sinh a\left(x_{j}-x_{s}\right)\right]^{-1} S_{n}^{(k)}(y) \exp \left[\sum_{s=1}^{n} x_{s}\left(i k_{s}-(n-1) a\right],\right.
$$

where $S_{n}^{(k)}(y)$ is a polynomial in $y_{s}=\exp \left(2 a x_{s}\right), \quad 1 \leqslant s \leqslant n$ and the degree of each variable $\left\{y_{s}\right\}$ in $S_{n}^{(k)}(y)$ does not exceed $n-1$.

As a consequence of $H_{s}^{(n)} \psi_{n}^{(k)}(x)=\frac{k^{2}}{2} \psi_{n}^{(k)}(x)$ and (6), we get an equation for $S_{n}^{(k)}(y)$,

$$
\begin{gathered}
4 a \sum_{j=1}^{n} y_{j} \frac{\partial}{\partial y_{j}}\left(y_{j} \frac{\partial S_{n}^{(k)}}{\partial y_{j}}\right)+\sum_{j \neq s}^{n} \frac{y_{j}+y_{s}}{y_{j}-y_{s}}\left[2 a\left(y_{s} \frac{\partial}{\partial y_{s}}-y_{j} \frac{\partial}{\partial y_{j}}\right)+\right. \\
\left.+i\left(k_{s}-k_{j}\right)\right] S_{n}^{(k)}+2\left\{\frac{a n(n-1)(2 n-1)}{3}+\sum_{j=1}^{n}\left[2 \left(i k_{j}-\right.\right.\right. \\
\left.\left.-a(n-1)) y_{j} \frac{\partial}{\partial y_{j}}-i k_{j}(n-1)\right]\right\} S_{n}^{(k)}=0 .
\end{gathered}
$$


Polynomial solutions of (7) exist if and only if for each pair of indices $(j, s), \quad 1 \leqslant j<s \leqslant n$ the polynomial

$$
\left[2 a\left(y_{s} \frac{\partial}{\partial y_{s}}-y_{j} \frac{\partial}{\partial y_{j}}\right)+i\left(k_{s}-k_{j}\right)\right] S_{n}^{(k)}
$$

will be divisible on $\left(y_{j}-y_{s}\right)$ without any rest. Let us represent $S_{n}^{(k)}(y)$ in the form

$$
S_{n}^{(k)}(y)=\sum_{\{m\} \in \mathbf{Z}^{n}} d_{m_{1} \ldots m_{n}}^{(k)} \prod_{\lambda=1}^{n}\left(y_{\lambda}\right)^{m_{\lambda}},
$$

where $d_{\{m\}}^{(k)}=0$ if at least one of the numbers $m_{s}$ does not belong to the segment $[0, n-1]$. Then the above condition can be expressed in the form of the system of $\frac{(n-1)(2 n-1) n^{n-1}}{2}$ linear equations for $d_{\{m\}}^{(k)}$,

$$
\sum_{l \in \mathbf{Z}} d_{m_{1} \ldots m_{j}+l \ldots m_{s}-l \ldots m_{n}}^{(k)}\left[m_{j}-m_{s}+2 l+\frac{i}{2 a}\left(k_{j}-k_{s}\right)\right]=0 .
$$

The substitution of (8) into (7) leads us to the equation

$$
\begin{aligned}
& \sum_{\{m\} \in \mathbf{Z}^{n}}\left(\prod_{\lambda=1}^{n} y_{\lambda}^{m_{\lambda}}\right) d_{\{m\}}^{(k)}\left\{\sum_{j=1}^{n}\left[2 m_{j}^{2}+\frac{2 i k_{j} m_{j}}{a}-\left(2 m_{j}+\frac{i k_{j}}{a}\right)(n-1)\right]-\right. \\
& \left.-\sum_{j \neq s}^{n} \frac{y_{j}+y_{s}}{y_{j}-y_{s}}\left[m_{j}-m_{s}+\frac{i}{2 a}\left(k_{j}-k_{s}\right)\right]+\frac{n(n-1)(2 n-1)}{3}\right\}=0 .
\end{aligned}
$$

With the use of (9) one can perform explicitly the division on $y_{j}-y_{s}$ in (10) and arrive, after comparison of the coefficients at various degrees of variables $\left\{y_{\lambda}\right\}$, to yet another system of $n^{n}$ linear equations for $d_{\{m\}}^{(k)}$,

$$
\begin{aligned}
d_{m_{1} \ldots m_{n}}^{(k)} & \left\{\sum_{j=1}^{n}\left[2 m_{j}^{2}+2 m_{j}\left(\frac{i k_{j}}{a}-n+1\right)-\frac{i k_{j}}{a}(n-1)\right]+\frac{n(n-1)(2 n-1)}{3}\right\}+ \\
+ & \sum_{j \neq s} \operatorname{sign}(l) \sum_{l \in \mathbf{Z}}\left[m_{s}-m_{j}+\frac{i\left(k_{s}-k_{j}\right)}{2 a}\right] d_{m_{1} \ldots m_{j}+l \ldots m_{s}-l \ldots m_{n}}^{(k)}=0 .
\end{aligned}
$$

The system of $\frac{\left(2 n^{2}-n+1\right)}{2} n^{n-1}$ Eqs. (9), (11) allows one to determine the coefficients $d_{\{m\}}^{(k)}$ up to a normalization constant.

Proposition 3. $S_{n}^{(k)}(y)$ is a homogeneous polynomial of degree $n(n-1) / 2$.

The proof is based on the system (9). Indeed, it follows from this system that $d_{\{m\}}^{(k)}=0$ if $m_{j}=m_{q}=0$ or $m_{j}=m_{q}=n-1$ for some pair of indices $(j, q)$. Let us consider an arbitrary coefficient $d_{m_{1} \ldots m_{n}}^{(k)}$ for which

$$
\mu(d)=\sum_{s=1}^{n} m_{s}<\frac{n(n-1)}{2} .
$$


In this case, among the numbers $\left\{m_{s}\right\}$ at least two coincide. Let $r$ be the minimal value of the corresponding $\left\{m_{s}\right\}$.

Let us choose among them an arbitrary pair $m_{j}=m_{q}=r$ and express $d_{m_{1} \ldots m_{j} \ldots m_{q} \ldots m_{n}}^{(k)}$ with the use of (9) through coefficients $d_{m_{1} \ldots m_{j-l} \ldots m_{q+l} \ldots m_{n}}^{(k)}$ with the same value of $\mu(d)$.

Repeating for these coefficients the same procedure, we shall finally find that the initially chosen coefficient $d_{m_{1} \ldots m_{n}}^{(k)}$ can be represented as a linear combination of those $\left\{d_{\{m\}}^{(k)}\right\}$ for which at least two from the numbers $m_{1}, \ldots m_{n}$ are zero. Hence, $d_{m_{1} \ldots m_{n}}^{(k)}=0$ as $\mu(d)<\frac{n(n-1)}{2}$. The case $\mu(d)>\frac{n(n-1)}{2}$ can be considered analogously.

Proposition 4. The coefficients $d_{\{m\}}^{(k)}$ considered as functions of $k$ and a are determined by the values of $n-1$ variables $\omega_{j}=\frac{k_{j}-k_{j+1}}{a}, \quad 1 \leqslant j \leqslant n-1$.

For the proof it is enough to establish that the coefficients in the system (11) depend on $k$ and $a$ only in the form of the combinations $\left\{\omega_{j}\right\}$. This can be done using the identities

$$
\begin{gathered}
\sum_{j=1}^{n} k_{j} m_{j}=n^{-1}\left(\frac{1}{2} \sum_{j \neq s}\left(k_{j}-k_{s}\right)\left(m_{j}-m_{s}\right)+\sum_{j=1}^{n} m_{j} \sum_{s=1}^{n} k_{s}\right), \\
2 \sum_{j=1}^{n} m_{j}^{2}=n^{-1}\left[\sum_{j \neq s}^{n}\left(m_{j}-m_{s}\right)^{2}+\left(\sum_{s=1}^{n} m_{s}\right)^{2}\right]
\end{gathered}
$$

and Proposition 3. Finally, the system (11) can be written as

$$
\begin{gathered}
\sum_{j<s}\left\{d_{\{m\}}^{(k)}\left[\frac{m_{j}-m_{s}}{n}\left(\frac{i}{a}\left(k_{j}-k_{s}\right)+m_{j}-m_{s}\right)+\frac{n+1}{6}\right]-\right. \\
\left.-\sum_{l \in \mathbf{Z}} \operatorname{sign}(l)\left[\frac{i}{2 a}\left(k_{j}-k_{s}\right)+m_{j}-m_{s}\right] d_{m_{1} \ldots m_{j}+l \ldots m_{s}-l \ldots m_{n}}^{(k)}\right\}=0 .
\end{gathered}
$$

Let $\{P\}$ be the following set of numbers $\left\{m_{s}\right\}: m_{j}=P j-1, \quad j=1, \ldots, n$, where $P$ is some permutation of the numbers $1, \ldots, n$.

Proposition 5. All coefficients $d_{\{m\}}^{(k)}$ which are different from zero and have some coinciding numbers within the values of $\left\{m_{s}\right\}$ are linear combinations of the coefficients $d_{\{P\}}^{(k)}$ which, in their values, are defined from the system (9) up to the normalization constant $d_{0}$ :

$$
d_{\{P\}}^{(k)}=d_{0} \prod_{\lambda<\mu}^{n}\left[1+\frac{i}{2 a}\left(k_{P^{-1} \lambda}-k_{P^{-1} \mu}\right)\right] .
$$

The first part of the statement is proved by using a scheme analogous to that of the proof of Proposition 3.

To prove (13), let us note that in the case where all $\left\{m_{s}\right\}$ are different the system (9) contains the subsystem of $\frac{(n-1)}{2} n$ ! equations

$$
\begin{gathered}
d_{m_{1} \ldots m_{j} \ldots m_{s} \ldots m_{n}}^{(k)}\left[-1+\frac{i}{2 a}\left(k_{j}-k_{s}\right)\right]+ \\
+d_{m_{1} \ldots m_{s} \ldots m_{j} \ldots m_{n}}^{(k)}\left[1+\frac{i}{2 a}\left(k_{j}-k_{s}\right)\right]=0, \quad m_{s}=m_{j}+1 .
\end{gathered}
$$


Let $P$ be the permutation $\left(j \rightarrow m_{j}+1\right), 1 \leqslant j \leqslant n$, and $R$ be a permutation of the numbers $j$ and $s$ which does not act on other numbers from 1 to $\mathrm{n}$.

The subsystem (14) can be written as

$$
d_{\{P\}}^{(k)}\left[1+\frac{i}{2 a}\left(k_{s}-k_{j}\right)\right]=d_{\{P R\}}^{(k)}\left[1+\frac{i}{2 a}\left(k_{j}-k_{s}\right)\right] .
$$

With the use of the condition $m_{s}=m_{j}+1$, let us represent (13) in the form

$$
\begin{gathered}
d_{\{P\}}^{(k)}=d_{0}\left[1+\frac{i}{2 a}\left(k_{j}-k_{s}\right)\right] \prod_{\lambda=1}^{m_{j}}\left[1+\frac{i}{2 a}\left(k_{P^{-1} \lambda}-k_{j}\right)\right]\left[1+\frac{i}{2 a}\left(k_{P^{-1} \lambda}-k_{s}\right)\right] \times \\
\times \prod_{\mu=m_{s}+2}^{n}\left[1+\frac{i}{2 a}\left(k_{j}-k_{P^{-1} \mu}\right)\right]\left[1+\frac{i}{2 a}\left(k_{s}-k_{P^{-1} \mu}\right)\right] \times \\
\times \prod_{\lambda<\mu}^{n}\left[1+\frac{i}{2 a}\left(k_{P^{-1} \lambda}-k_{P^{-1} \mu}\right)\right] . \\
\lambda, \mu \neq m_{j}+1, m_{s}+1 .
\end{gathered}
$$

The corresponding expression for $d_{\{P R\}}^{(k)}$ differs from (16) only by the change $k_{j} \leftrightarrow k_{s}$.

Hence, Eqs. (15) are valid for all $P \in \pi_{n}$.

The formula (13) allows one to determine the first terms in asymptotic expansions of the function $\phi_{n}^{(k)}(x)(6)$.

Proposition 6. If $x_{P(\lambda+1)}-x_{P \lambda} \rightarrow \infty, \quad 1 \leqslant \lambda \leqslant n-1$, then

$$
\lim \phi_{n}^{(k)}(x) \exp \left[-i \sum_{\lambda=1}^{n} k_{\lambda} x_{\lambda}\right]=2^{\frac{n(n-1}{2}}(-1)^{P} d_{\left\{P^{-1}\right\}}^{(k)},
$$

where $(-1)^{P}$ is the parity of the permutation $P$.

Let us formulate the scheme of the proof for the case $x_{\lambda+1}-x_{\lambda} \rightarrow+$ infty, $\quad 1 \leqslant \lambda \leqslant n-1$.

Under these conditions,

$$
\left.\prod_{\lambda>\mu}^{n} \sinh ^{-1} a\left(x_{\lambda}-x_{\mu}\right)\right] \sim 2^{\frac{n(n-1)}{2}} \exp \left[-\sum_{\lambda=1}^{n} x_{\lambda}(n-2 \lambda+1)\right]
$$

and

$$
\begin{gathered}
\phi_{n}^{(k)}(x) \exp \left[-i \sum_{\lambda=1}^{n} k_{\lambda} x_{\lambda}\right] \sim \\
\sim 2^{\frac{n(n-1}{2}} \sum_{\{m\}} d_{\{m\}}^{(k)} \exp \left[-2 a \sum_{\lambda=1}^{n-1}\left(x_{\lambda+1}-x_{\lambda}\right) f_{\lambda}(\{m\})\right],
\end{gathered}
$$

where $f_{\lambda}(\{m\})=\sum_{r=1}^{\lambda}\left(m_{r}-r+1\right)$.

For the sets $\{m\}$ to which nonzero $d_{\{m\}}^{(k)}$ correspond, $f_{\lambda}(\{m\} \geqslant 0$. The equality here takes place if $m_{\lambda}=\lambda-1, \quad 1 \leqslant \lambda \leqslant n$. The case of arbitrary permutation $P \in \pi_{n}$ can be considered in a completely analogous way. 
Notation. If the constant $d_{0}$ in (13) is chosen in the form

$$
d_{0}=\prod_{j=1}^{n-1}(2 j+1) ! \prod_{\lambda>\mu}^{n}\left[\frac{i}{a}\left(k_{\lambda}-k_{\mu}\right)\right]^{-1} \prod_{\lambda \neq \mu}^{n}\left[1+\frac{i}{a}\left(k_{\lambda}-k_{\mu}\right)^{-1}\right]
$$

one gets

$$
d_{\{P\}}^{(k)}=(-1)^{P} c\left(a^{-1} k_{P^{-1} 1}, \ldots a^{-1} k_{P^{-1} n}\right),
$$

where $c\left(k_{1}, \ldots k_{n}\right)$ is the Harish-Chandra function for symmetric space $X_{n}$. This fact can be easily established by comparison of (13) and explicit expression of the c-function by Euler Bfunctions. In accordance with Proposition 6, the ratios of the coefficients (13) determine the $S$-matrix of many-body quantum scattering for the Sutherland problem at a specific value of the coupling constant [7].

Note also that, in accordance with Proposition 5, the solutions of the system (9) must satisfy Eqs. (12), i.e., the system (12) is a consequence of (9). It would be of interest to find a direct algebraic proof of this fact.

\section{Examples of explicit calculations of the d-coefficients}

Let us introduce the following notation: let $\left[\lambda_{1} \ldots \lambda_{n}\right]$ be the permutation $\left(1 \rightarrow \lambda_{1}, \ldots, n \rightarrow\right.$ $\left.\lambda_{n}\right) ; \quad r_{\lambda \mu}=\frac{i}{2 a}\left(k_{\lambda}-k_{\mu}\right)$.

1. $n=3$. In accordance with (13), the coefficients $d_{\{P\}}^{(k)}$ have the form

$$
\begin{array}{ll}
d_{012}^{(k)}=d_{0}\left(1+r_{12}\right)\left(1+r_{13}\right)\left(1+r_{23}\right), & d_{102}^{(k)}=d_{0}\left(1+r_{21}\right)\left(1+r_{23}\right)\left(1+r_{13}\right), \\
d_{210}^{(k)}=d_{0}\left(1+r_{32}\right)\left(1+r_{31}\right)\left(1+r_{21}\right), & d_{021}^{(k)}=d_{0}\left(1+r_{13}\right)\left(1+r_{12}\right)\left(1+r_{32}\right), \\
d_{120}^{(k)}=d_{0}\left(1+r_{31}\right)\left(1+r_{32}\right)\left(1+r_{12}\right), & d_{201}^{(k)}=d_{0}\left(1+r_{23}\right)\left(1+r_{21}\right)\left(1+r_{31}\right) .
\end{array}
$$

The single nonzero coefficient which does not coincide with $d_{\{P\}}^{(k)}$ is $d_{111}^{(k)}$. It is determined from (9) at $m_{1}=0, m_{2}=1, m_{3}=2, j=1, s=3$ :

$$
d_{111}^{(k)}=d_{0}\left[6-r_{12}^{2}-r_{13}^{2}-r_{23}^{2}\right]
$$

2. $n=4$. In addition to 24 coefficients $d_{\{P\}}^{(k)}$, yet another 14 coefficients are different from zero. They can be naturally divided into three sets. The first two sets contain coefficients with three coinciding lower indices.

Their calculation is completely analogous to the calculation of $d_{111}^{(k)}$ at $n=3$,

$$
\begin{aligned}
& d_{1113}^{(k)}=d_{0}\left(1+r_{14}\right)\left(1+r_{24}\right)\left(1+r_{34}\right)\left(6-r_{12}^{2}-r_{13}^{2}-r_{23}^{2}\right), \\
& d_{2220}^{(k)}=d_{0}\left(1+r_{41}\right)\left(1+r_{42}\right)\left(1+r_{43}\right)\left(6-r_{12}^{2}-r_{13}^{2}-r_{23}^{2}\right) .
\end{aligned}
$$

Other coefficients of these sets $d_{1131}^{(k)}, d_{1311}^{(k)}, d_{3111}^{(k)}, d_{2202}^{(k)}, d_{2022}^{(k)}, d_{0222}^{(k)}$ are obtained from (17) and (18) correspondingly by permutations of indices [1243], [1342], [2341] in $\left\{r_{\lambda \mu}\right\}$. 
The third set contains coefficients with two pairs of coinciding indices: $d_{1122}^{(k)}, d_{2211}^{(k)}, d_{2112}^{(k)}$, $d_{1221}^{(k)}, d_{1212}^{(k)}, d_{2121}^{(k)}$. All of them can be determined from Eqs. (9) since the coefficients of the first set are already known. So, putting in (9) $m_{1}=m_{2}=m_{3}=1, m_{4}=3 ; j=3, s=4$, one gets

$$
d_{1113}^{(k)}\left(-2+r_{34}\right)+d_{1122}^{(k)} r_{34}+d_{1131}^{(k)}\left(2+r_{34}\right)=0,
$$

which, after rather simple calculations, allows one to find $d_{1122}^{(k)}$ :

$$
\begin{gathered}
d_{1122}^{(k)}=d_{0}\left[18+\left(r_{13}+r_{24}\right)\left(14-2\left(r_{13}+r_{24}-r_{13}^{2}-r_{24}^{2}-\right.\right.\right. \\
\left.-3\left(r_{12}^{2}+r_{34}^{2}\right)-r_{12} r_{34}+r_{12}^{2} r_{34}^{2}\right)-\left(r_{12}^{2}+r_{34}^{2}\right)\left(6+r_{13} r_{24}-r_{12} r_{34}\right)- \\
\left.-r_{13} r_{24}\left(r_{13}^{2}+r_{24}^{2}\right)\right] .
\end{gathered}
$$

Other five coefficients of this set can be found with the use of (20) by permutations [3412], [3214], [4123], [1324], [4213] of the indices in $\left\{r_{\lambda \mu}\right\}$.

3. At $n=5$ there are 171 coefficients $d^{(}(k)_{\{m\}}$ which differ from zero and have coinciding indices.

They are divided into 8 sets; at $n=6$ there are 2112 such coefficients and the number of sets grows to 21 . The algorithm of their construction where $d_{\{P\}}^{(k)}$ are supposed to be known can be simply realized on a computer.

The explicit expressions for zonal spherical functions $\Phi_{n}^{(k)}(x)$ are obtained by symmetrization of the corresponding singular eigenfunctions $\phi_{n}^{(k)}(x)$ of the Sutherland operators.

\section{References}

[1] Helgason, S., Differential Geometry and Symmetric Spaces, New York: Academic Press, 1962.

[2] Vilenkin, N. Ja., Special Functions and the Theory of Group Representations, Moscow: Nauka, 1965 (Russian).

[3] Vretare, L., Formulas for Elementary Spherical Functions and Generalized Jacobi Polynomials, SIAM J. Math. Anal., 1984, vol. 15, pp. 805-834.

[4] Anderson, A. and Camporesi, R., Intertwining Operators for Solving Differential Equations with Applications to Symmetric Spaces, Commun. Math. Phys., 1990, vol.130, no. 1, pp. 61-82.

[5] Sekiguchi, J., Zonal Spherical Functions on Some Symmetric Spaces, Publ. RIMS. Kyoto Univ., 1977, Suppl. 12, pp. 455-464.

[6] Gindikin, S. G. and Karpelevich, F. I., Plancherel Measure for Symmetric Riemannian Spaces of NonPositive Curvature, Dokl. Akad. Nauk SSSR, 1962, vol.145, no. 2, pp. 252-255 (Russian).

[7] Olshanetsky, M. A. and Perelomov, A. M., Quantum Integrable Systems Related to Lie Algebras, Phys. Rep., 1983, vol. 94, no. 6, pp. 313-404.

[8] Chalykh, O.A. and Veselov, A.P., Commutative Rings of Partial Differential Operators and Lie Algebras, Comm. Math. Phys., 1990, vol. 126, no. 3, pp. 597-611.

[9] Inozemtsev, V.I., Integrable Heisenberg-Dirac Chains with Variable Range Exchange: Integrable Quantum Spin Chains, Saarbrücken: Lambert, 2013. 